\title{
Akkumulation von Wissen über eine naturnahe Landschaft (San Ynez Valley, Kalifornien)
}

\section{Einleitung}

Die Mehrzahl der empirischen Untersuchungen, die sich mit der Wahrnehmung und dem Wissen von Individuen oder Gruppen über die Umwelt befassen, beschäftigen sich mit städtischen oder bebauten Milieus (z. B. APPLEYARD 1970, APPLEYARD 1979, EVANS 1980). Aus der Zusammenarbeit von Architekten, Psychologen, Planern und Geographen ist in den letzten Jahrzehnten eine Vielzahl von Arbeiten entstanden, die sich bei der Gestaltung und Planung bebauter Umwelten verwenden lassen (MOORE und GOLLEDGE 1976).

Dabei steht in solchen Untersuchungen zunehmend weniger die Analyse der «Produkte» oder Konstrukte (z. B. mental maps, kognitive Karten, Umweltwissen) als die Analyse der Prozesse der Wissensakkumulierung im Vordergrund (vgl. GOLLEDGE et al. 1985). Auch diese neueren Arbeiten, die in der Geographie noch kaum Beachtung finden, beziehen sich fast ausschließlich auf die Bedingungen in städtischen Milieus.

Ziel dieses Artikels ist es, einige Ergebnisse aus einer empirischen Untersuchung über die Akkumulation von Wissen über eine naturnahe Landschaft, die nur wenig durch menschliche Tätigkeiten gestaltet ist, darzustellen und in einem größeren Zusammenhang der gesellschaftlichen Entwicklung zu diskutieren. Insbesondere geht es um die Analyse von Bedingungen des Wissenserwerbes. Ausgangspunkt ist dabei die Vermutung, daß sich gerade in naturnahen Landschaften, die a priori wenig anthropogenen Symbolgehalt aufweisen, idealtypische Voraussetzungen für das Studium dieser Prozesse finden. Implizit wird also davon ausgegangen, daß der konkrete Wahrnehmungszusammenhang und die realräumlichen Voraussetzungen zumindest relevante Bedingungen für den Prozeß des Wissenserwerbes sind (vgl. auch woHLwILL 1976).

Im Vordergrund der folgenden Überlegungen steht die Frage nach den Strukturen, durch die Menschen unmittelbar und bewußt Landschaften erkunden, sich im Laufe der Zeit reproduzierbares Wissen über diese Landschaften aneignen und damit eine Beziehung zur Umwelt aufbauen (im Sinne von «Place» by RELPH 1976). Das Interesse gilt damit weniger der umweltpsychologischen Theoriebildung als der humanökologischen Interpretation der Ergebnisse.

Als Untersuchungsgebiet wurde dabei eine nahezu unbesiedelte, naturnahe und praktisch nur zur Erholung genutzte bewaldete Landschaft im Hinterland von Santa
Barbara (Los Padres Forest) ausgewählt. Zur Erläuterung einiger Begriffe aus der kognitiven Wissenschaft, die in der Humanökologie zunehmend beachtet wird (z. B. KAPLAN Und KAPLAN 1982; BERRY 1976), wird das Modell von CHAFE (1977) eingeführt.

\section{Kognitives Modell}

Zur Darstellung des Verhältnisses von Begriffen wie Bewußtsein, Gedächtnis oder Wahrnehmung, die in Studien über Umweltwissen einen zentralen Stellenwert einnehmen, eignet sich das relativ einfache und durchaus operationelle Modell des Linguisten CHAFE (1977), der sich insbesondere mit den Phänomenen der Wiedergabe und Verbalisierung von "past experience» (Erlebnissen aus der Vergangenheit) beschäftigte. Abb. 1 zeigt das Modell im Überblick.

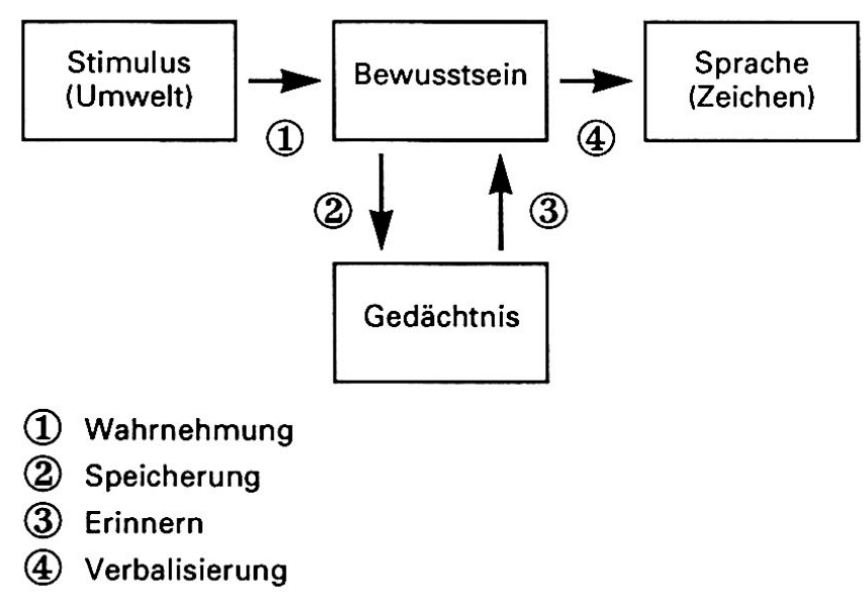

Abb. 1 Systemmodell nach CHAFE (1977).

CHAFE (1977) spricht von einem System mit den Elementen Stimulus (Umwelt), Bewußtsein, Gedächtnis und Sprache. Umwelt kann in dieser Darstellung als Stimulus oder in den Worten von CHAFE (1977) präziser als «outside world» aufgefaßt werden. Das Bewußtsein stellt eine Art von Glaube («belief») über die Phänomene der Um-

Pierre Walther, Dr., Infraconsult AG, Bitziusstr. 40, 3006 Bern 
welt dar und ist bezüglich seiner Aufnahmekapazität begrenzt.

Im Modell von CHAFE (1977), das sich in der psychologischen Interpretation linguistischer Phänomene bewährt hat, werden Wahrnehmung, Speicherung («storage»), Erinnern («recall») und Verbalisierung als die wesentlichen Prozesse verstanden. Das Erinnern kann durch Stimuli wesentlich erleichtert werden. Zum Beispiel ist das «freie Erinnern» schwieriger als das Wiedererkennen («recognition»).

CHAFE (1977) hat sich vor allem interessiert, wie Phänomene, die entweder als Erinnerung oder durch Wahrnehmung im Bewußtsein vorhanden sind, mittels Verbalisierung oder durch die Anwendung anderer Sprachen (z. B. Körpersprache, Zeichnungen) gleichsam in Sprache verpackt werden. Diesem Prozeß ist aus methodischer Sicht in empirischen Erhebungen zum Umweltwissen genügend Beachtung zu schenken.

\section{Bisherige Untersuchungen}

Im Vordergrund dieser Untersuchung steht das Wissen über die Umwelt, das im Langzeitgedächtnis festgehalten wird und im Rahmen eines Interviews wiedergegeben werden kann («recall» und Verbalisierung). Im folgenden sollen aus der Vielzahl von Studien kurz einige Erkenntnisse zusammengefaßt werden (vgl. auch EVANS 1980; KAPLAN 1982).

EVANS (1980) hat darauf hingewiesen, daß die Prozesse der Wahrnehmung und Speicherung von Informationen unter realen Umweltbedingungen (z. B. Städte, Landschaften) anders verlaufen als unter Laborbedingungen. Ergebnisse aus psychologischen Labortests sind daher nur beschränkt auf die im folgenden dargestellten Untersuchungen anwendbar.

Zentral ist dabei, daß die Umwelt als Stimulus aus bedeutungsvollen Objekten besteht und daß die Bedeutungen im Kontext erfaßt werden. Gerade in städtischen Umwelten prägen diese semantischen Zusammenhänge den Prozeß des Wissenserwerbes (vgl. APPLEYARD 1979). EVANS (1980) weist darauf hin, daß diesen Zusammenhängen in der Forschung bisher zu wenig Beachtung geschenkt worden ist.

BECK und wOOD (1976) versuchten, Umwelt als Informationsfeld zu verstehen, aus dem durch Wahrnehmung Informationen extrahiert und in Wissen transformiert wird. Nach ihrer Ansicht spielt die "Lesbarkeit» der Landschaft bei der Akkumulation von Umweltwissen eine bedeutende Rolle. In der Regel sind gestaltete Umwelten besser lesbar als naturnahe Landschaften.

In ihren Untersuchungen haben SAAB et al. (1984) auf die Bedeutung der Attraktivität und Sichtbarkeit der Umweltobjekte für die Wahrnehmung und Speicherung der Informationen hingewiesen. Aus kognitiven Untersuchungen (Schemata Theorie) geht auch hervor, daß sich Informanten besser an Unerwartetes erinnern als an das Selbstverständliche (BARONI et al. 1980).
GOLLEDGE et al. (1985) haben die Akkumulation von Umweltwissen in einer detaillierten Studie verfolgt. Sie kommen unter anderem zum Schluß, daß an Orten, an denen beim Erleben der Landschaft Entscheide (z. B. über Richtungsänderungen) gefällt werden müssen, mehr Umweltwissen akkumuliert wird. Mit zunehmender Vertrautheit mit einer Landschaft wird das Umweltwissen generalisiert.

\section{Anlage der empirischen Studie (Los Padres National Forest)}

Ziel dieser empirischen Untersuchung war es, mittels einer einfachen Methodik von Informanten, die einen typisierbaren Erlebnisbezug zu einer weitgehend naturnahen Landschaft aufweisen, Umweltwissen über diese Landschaft zu extrahieren. Dabei ging es nicht nur um die Dokumentation des "Produktes» (Umweltwissen), sondern auch um die Beobachtung des Prozesses der Wiedergabe dieses Wissens und um die Entwicklung einer Methode für "rapid environmental appraisal», die zum Beispiel zur Erarbeitung von Planungsgrundlagen eingesetzt werden könnte.

Als Untersuchunsgebiet wurde ein Ausschnitt aus dem Los Padres National Forest im Hinterland von Santa Barbara in Kalifornien, der durch eine kleine Straße sowie durch Wanderwege erschlossen ist, ausgewählt (vgl. Abb. 2, 3 und 4). Abb. 2 vermittelt einen Eindruck von der Naturnähe der Landschaft.

\subsection{Untersuchungskonzept}

Grundlage der empirischen Erhebung bildete eine Luftbildvergrößerung des Untersuchungsgebietes aus dem Gebiet von Los Padres (Abb. 4 und 5). Das Luftbild wurde verwendet zur Kommunikation über diese naturnahe Landschaft, in der nur wenige anthropogene Strukturen (kleine Straße im Talgrund, einige Campingplätze, Headquartier des Forstdienstes) erkenntlich sind. Für die Datenerhebung und die Unterstützung der Theoriebildung wurden zwei Gruppen von Informanten gebildet, bei denen angenommen werden konnte, daß sie einen unterschiedlichen Zugang und damit verschiedenes Erfahrungswissen über das naturnahe Untersuchungsgebiet besaßen. In jeder Gruppe befanden sich sieben Informanten.

Bei Gruppe 1 handelte es sich um Naturfreunde, die über den Sierra Club kontaktiert wurden und in der Agglomeration von Santa Barbara wohnten (etwa $40 \mathrm{~km}$ vom Untersuchungsgebiet des Los Padres Forest). Diese Informanten nutzten den Los Padres Forest gelegentlich an Wochenenden für die Erholung (gelegentliche Besuche oder Camping).

Durch Vermittlung des US Forestry Service wurde eine zweite Gruppe von Informanten kontaktiert (Gruppe 2). Bei dieser Gruppe handelte es sich um Mitarbeiter des Forstdienstes, die seit Jahren im Untersuchungsgebiet 


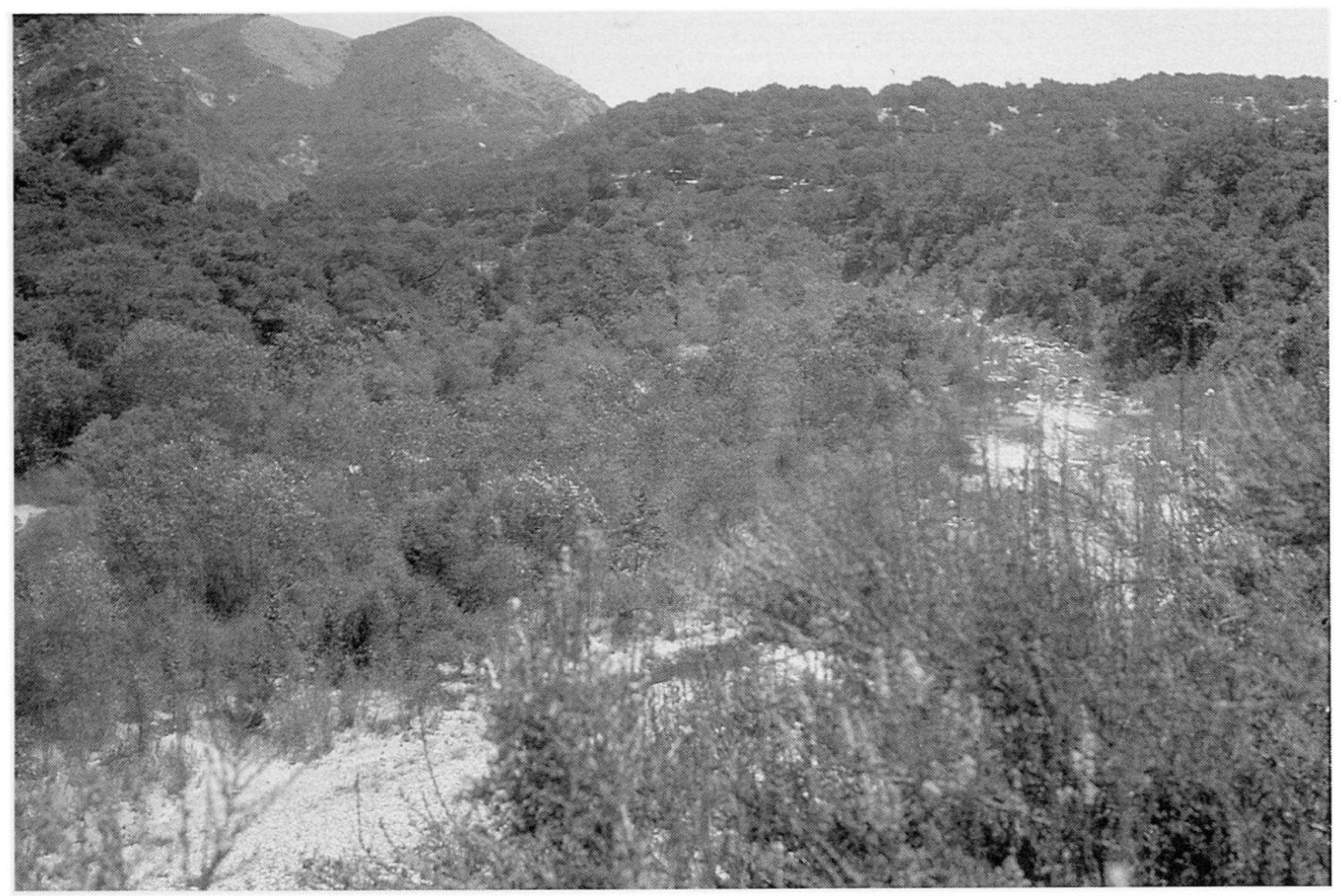

Abb. 2 Typischer Ausșchnitt aus dem Untersuchungsgebiet.

arbeiten (Brandbekämpfung, Überwachung der Campingplätze und Wanderwege). Wie sich herausstellte, wohnten zwei der sieben Informanten auch im Untersuchungsgebiet (einfache Wohnwagenunterkünfte). Das Hauptquartier des Forstdienstes befindet sich im Untersuchungsgebiet.

Mit Absicht wurden die Gruppen relativ klein gewählt. Dies erlaubte, mittels qualitativer Methoden einen vertieften Einblick in die Beziehung des Informanten zum Untersuchungsgebiet zu gewinnen (mittels Methoden der "oral history»), was für die Interpretation der gewonnenen Informationen sehr wichtig war.

\subsection{Methodik, Datenaufnahme}

Die Informanten wurden einzeln entweder zu Hause oder am Arbeitsplatz interviewt. Zuerst wurde dabei die Kompetenz der einzelnen Informanten und damit die Homogenität der Gruppen bezüglich Interessen, Vertrautheit mit dem Gebiet, Kenntnissen und Aktivitätsräumen (durch Zeichnen von Karten des Aktivitätsraumes) überprüft. Dieser Teil bildete die Einleitung der 3bis 4stündigen Interviews.

Zur Datengewinnung wurde über die Luftbildvergrößerung (1:10 000) ein Koordinatennetz mit Quadraten von je $10 \mathrm{~cm}$ Seitenlänge gelegt. Ziel des Interviews war es, schrittweise von Quadrat zu Quadrat gehend möglichst viele Objekte, die im Quadrat vorkommen, mit möglichst präzisen Begriffen zu benennen. Das Koordinatennetz erfüllt dabei eine wichtige Funktion (geführte Kommunikation, Abtasten des ganzen Raumes nach Informationen).

Die Informationsaufnahme in einem Quadrat wurde abgebrochen, wenn der Informationseinfluß über längere Zeit gestoppt schien und bei den Informanten ein deutliches Abflauen der Aufmerksamkeit zu spüren war.

Nach dieser Methode schien den Informanten der Prozeß der «Verbalisierung» der Informationen (vgl. obige Diskussion des Modelles von CHAFE 1977) einfach. Anders als in anderen Studien, in denen etwa mit Zeichnungen zur Umwelt gearbeitet wird (z. B. in MOORE und GOLLEDGE 1975), beschränkte sich der Prozeß auf ein bloßes Antippen der Objekte auf dem Bild und auf das Nennen von Begriffen.

Bei der Ausarbeitung dieser Methode stützten wir uns auf die Ergebnisse älterer Studien (z. B. BLAUT et al. 1970; GOSSEN 1974), in denen nachgewiesen worden war, daß sich Luftbilder als Medium (oder Stimulus) für die Extraktion von Umweltwissen eignen. Alle Informanten waren fähig, das Luftbild zu lesen und die komplexen 


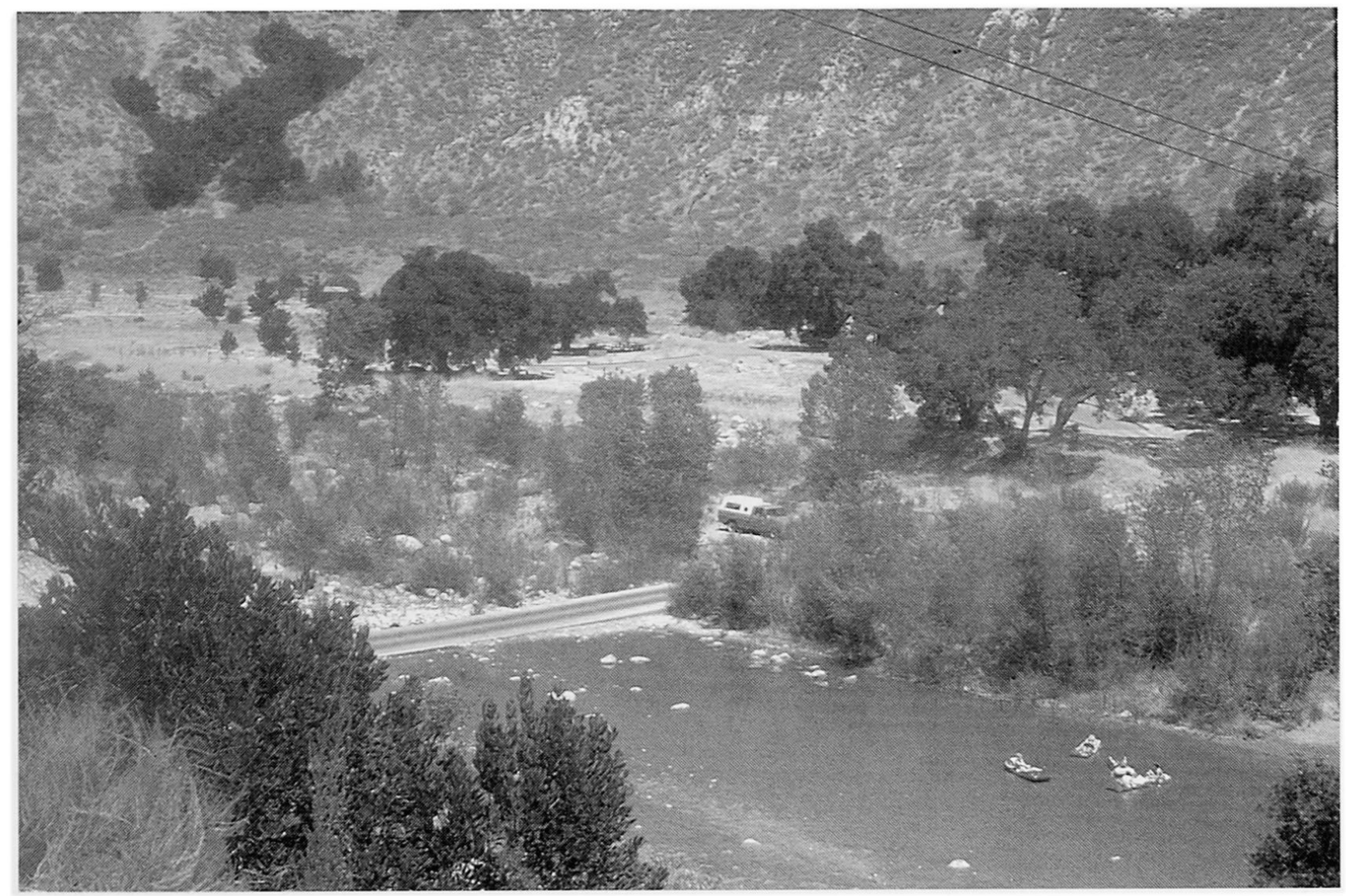

Abb. 3 Campingplatz im Los Padres National Forest.

geometrischen Transformationen von der erlebten Welt zur Perspektive der Luftaufnahme mühelos zu vollziehen.

Generell hat sich diese Methode bewährt und stieß auch bei den Informanten auf Interesse, so daß die Atmosphäre während der Interviews entspannt und produktiv war. In allen Fällen waren die Sitzungen angeregt, und die Informanten hatten Spaß, das Luftbild zu interpretieren.

\subsection{Auswertungen}

Alle Informationen wurden auf Tonband festgehalten und durch den Untersuchungsleiter schriftlich in einem Protokoll während des Interviews festgehalten und kommentiert. Die Tonbandprotokolle erlaubten eine relativ genaue Beobachtung des Wiedergabeprozesses (z. B. Analysen zu Verzögerungen in der Wiedergabe, zur Spontaneität der Nennungen, zu Assoziationsketten). Die Informationen (über 500 Nennungen) wurden anschließend vom Tonband in ein Protokoll übertragen und nach Kriterien klassiert (z. B. sichtbar/nicht sichtbar auf dem Lufbild, Genauigkeit und Sachbereich der Information, anthropogen/nicht anthropogen). Die räumlichen Daten (Objekte) wurden digitalisiert, was eine flexible
Auswertung der Daten mit Hilfe eines geographischen Informationssystems (ArcInfo) ermöglichte.

Aus methodischer Sicht erlaubte vor allem das Kriterium, ob das Objekt auf dem Luftbild sichtbar war oder nicht, aussagekräftige Auswertungen. Mit Hilfe dieses Kriteriums konnte zum Beispiel das Umweltwissen, dessen Wiedergabe durch den Stimulus (Luftbild) angeregt und damit mitgeprägt worden war, vom Umweltwissen, das auf direkter Erfahrung stammte, getrennt werden (insgesamt 32\% der Nennungen aus dieser Kategorie).

\section{Untersuchungsergebnisse}

Ziel dieses Kapitels ist es, einige Ergebnisse aus dieser empirischen Untersuchung zusammenzufassen. An einzelnen Beispielen kann auf Unterschiede gegenüber den Ergebnissen von Untersuchungen in städtischen Milieus (vgl. etwa MOORE und GOLLEDGE 1976) eingegangen werden.

Insgesamt zeigte sich, daß zwischen den Gruppen erhebliche Unterschiede bezüglich Aktivitätsraum und Interessen am Untersuchungsgebiet bestanden, doch daß die Homogenität innerhalb der Gruppen relativ groß war. 
Generell wurde aus den Resultaten die Individualität der Erfahrung der Landschaft und damit des Umweltwissens sichtbar (vgl. KAPLAN und KAPLAN 1982). Wie schon früher festgehalten wurde (z. B. in MOORE und GOLLEDGE 1976), ist es schwierig zu generalisieren. Der Psychologe und Pragmatiker JAMEs (1909) prägte schon zu Beginn dieses Jahrhunderts den Begriff des «pluralistic universe».

Es bestätigte sich insbesondere, wie stark das Wissen über eine naturnahe Umwelt, über die wenig bis keine Informationen (z. B. Presse, Filme) verfügbar sind, geprägt und gleichsam gebunden ist an:

- Aktivitätsraum (Wege als Grundstrukturen der Erkundung der Landschaft)

- Motive, Handlungen in der Landschaft

Über 75\% der genannten Objekte befinden sich in einer Distanz von weniger als $10 \mathrm{~m}$ vom Aktivitätsraum (Wege, die selbst abgeschritten oder abgefahren wurden). Über $80 \%$ der Nennungen mit hohem Detaillierungsgrad (z. B. Baumarten) finden sich unmittelbar neben den zu Fuß erwanderten Pfaden oder Orten. Dieses Resultat spiegelt unter anderem die speziellen Verhältnisse im Untersuchungsgebiet wieder, in dem wegen der dichten Bewaldung wenig bis keine Fernsicht möglich ist.

Das Umweltwissen von Gruppe 1 (Touristen) konzentriert sich auf den Bereich der touristischen Infrastrukturanlagen wie Campingplätze und Wanderwege. Der Aktivitätsraum von Gruppe 2 (Mitarbeiter des Forstdienstes) erwies sich als viel ausgedehnter (z. B. regelmäBiges Abschreiten der Schneisen im Wald, die gegen die Ausbreitung von Waldbränden geschlagen werden).

In der Erfahrung einer naturnahen Landschaft scheinen zudem anthropogene Objekte, die für Tätigkeiten symbolisch sind (APPLEYARD 1979) und die einen direkten Bezug zu Handlungen aufweisen, eine besondere Rolle zu spielen. Nur gerade $7 \%$ der Nennungen betrafen reine Naturobjekte. 62\% der Nennungen betrafen Infrastrukturobjekte wie beispielsweise Straßen, Brücken oder WC-Häuschen auf Campingplätzen (Abb. 3).

Insgesamt fiel auf, daß Mitarbeiter des Forstdienstes (Gruppe 2), die einen hohen Berührungsgrad mit dem Untersuchungsgebiet aufweisen, ein viel größeres De-

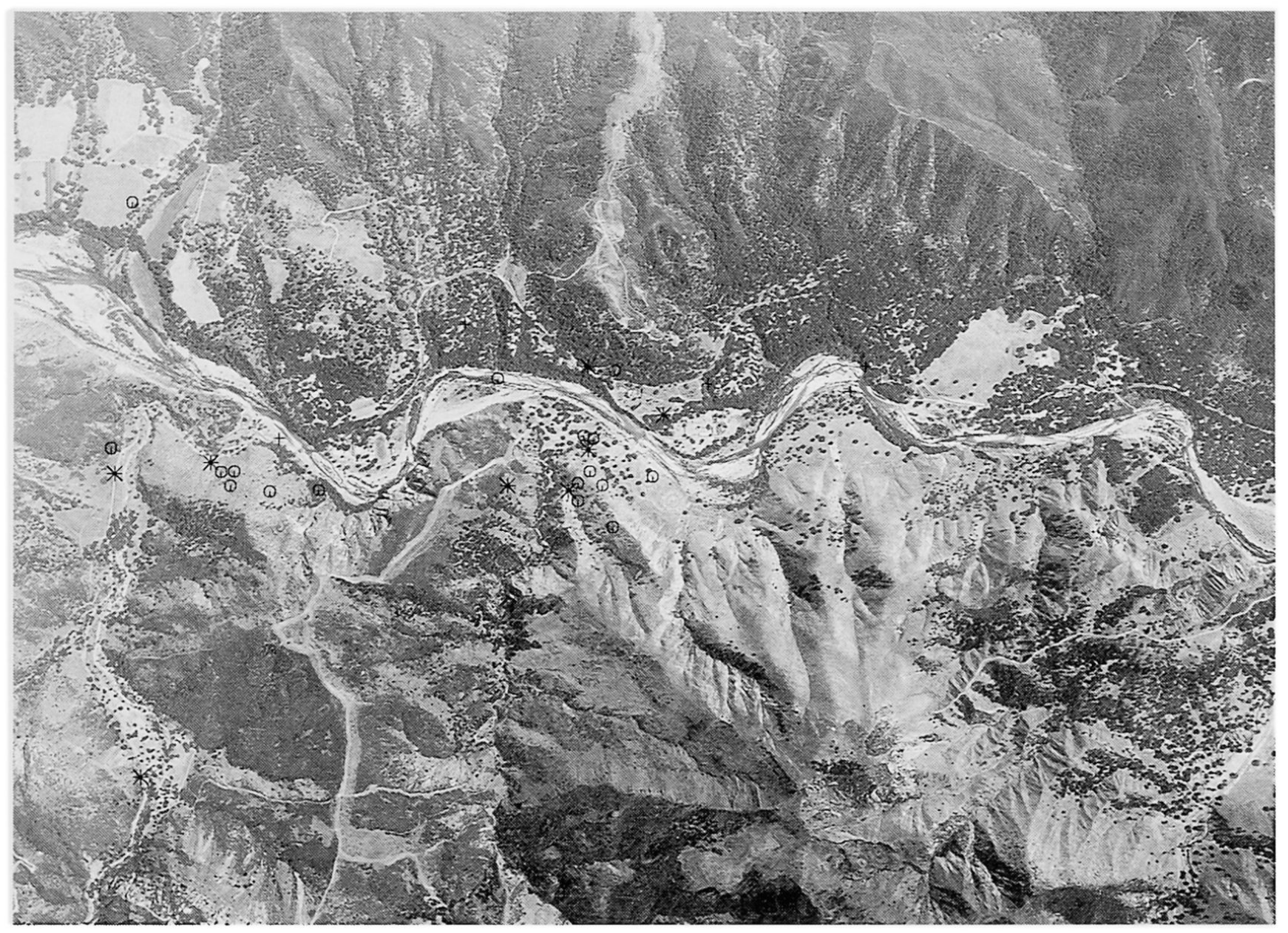

Abb. 4 Umweltwissen einer Informantin, die sich gelegentlich an Wochenenden im Untersuchungsgebiet aufhält (M ca. 1: 20 000; Kreuz = Objekt auf Luftbild klar sichtbar, Kreis = auf Luftbild schwach sichtbar, Stern = auf Luftbild nicht sichtbar). 
tailwissen über diese naturnahe Landschaft haben als die Touristen. Bei 83\% der Nennungen dieser Gruppe 2 handelt es sich um Detailwissen aus direkter Erfahrung (gegenüber $53 \%$ in Gruppe 1 der Touristen).

Entlang der Zufahrtstraße, wo Umweltwissen aus dem fahrenden Auto gesammelt wird, erwies sich der Detaillierungsgrad als klein, obwohl die Strecke allen Informanten bekannt war. Von praktisch allen Informanten wurden die zwei Brücken, die zumindest in einem Fall auf dem Luftbild nur schlecht sichtbar waren, spontan genannt. Eine ähnliche Feststellung hatten CARR und SCHISSLER (1969) in ihren detaillierten Untersuchungen gemacht.

Die Komplexität der sozial geprägten Bedingungen der Landschaftserfahrung zeigt sich im Umweltwissen auf Stufe Individuum. Abb. 4 zeigt das wiedergegebene Wissen durch eine Frau, die sich 2-bis 3 mal im Jahr zur Erholung im Untersuchungsgebiet aufhält. Das spezifische Umweltwissen, das die auf dem Luftbild nicht sichtbaren Objekte betrifft und damit allein aus dem Gedächtnis wiedergegeben ist, beschränkt sich auf Infrastrukturen im Bereich der beiden Campingplätze, auf denen sich die Informantin jeweils aufhält.

Die Information ist oft idiosynkratisch und an Erlebnisse geknüpft (eventuell ein Hinweis auf die Bedeutung des episodischen Gedächtnisses und ein Ansatz zu einer schemata-theoretischen Interpretation). Zum Beispiel erinnerte sie sich an eine Telephonkabine, die sie einmal in einer Notsituation für einen Anruf benützen mußte. Sie erinnerte sich, daß der Baum, unter den sie vor Jahren einmal das Zelt gestellt hatte, eine Eiche war.

Demgegenüber war das Umweltwissen eines Informanten aus Gruppe 2, der Mitarbeiter im Forstdienst ist und seit zwei Jahren im Untersuchungsgebiet einen Wohnwagen bewohnt, sowohl räumlich als auch thematisch und bezüglich Detaillierungsgrad ungleich vielfältiger (Abb. 5). Der Informant konnte zahlreiche Informationen zum Beispiel über Bademöglichkeiten im Fluß oder über die Eignung des Gewässers zum Fischen wiedergeben. Zudem kannte er den Standort jedes Abfalleimers, die er wöchentlich einmal zu leeren hatte.

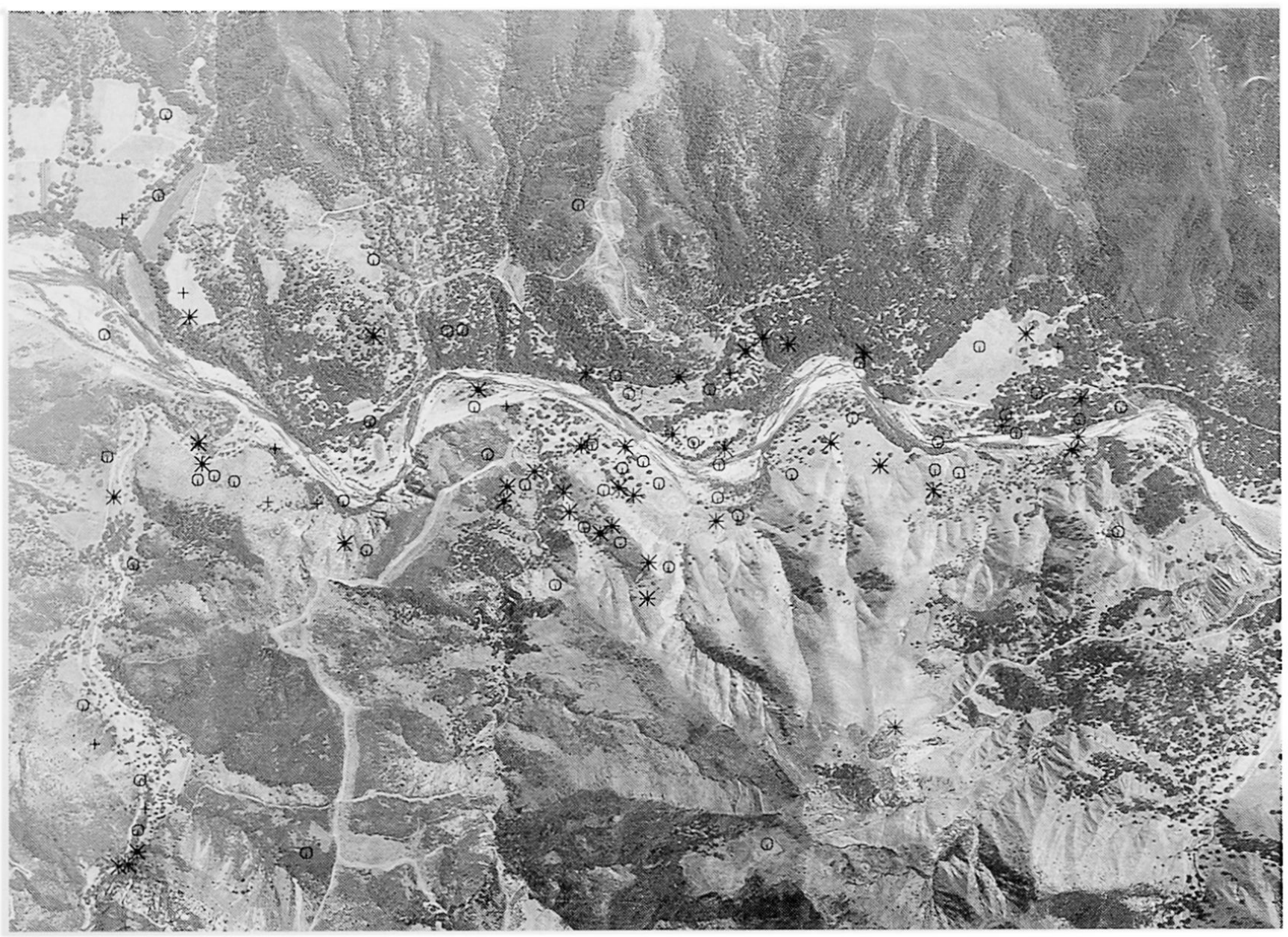

Abb. 5 Umweltwissen eines Informanten, der im Untersuchungsgebiet wohnt und arbeitet (M ca. 1:20000; Kreuz = Objekt auf Luftbild klar sichtbar, Kreis = auf Luftbild schwach sichtbar, Stern = auf Luftbild nicht sichtbar). 


\section{Diskussion}

Obwohl in dieser empirischen Untersuchung die emotionale Komponente nicht berücksichtigt wurden, vermittelt die Studie weitere Kenntnisse zum Verständnis der Prozesse der alltäglichen Erfahrung der naturnahen Umwelt durch Individuen und Gruppen. Diese Erkenntnisse sind in der humanökologischen Interpretation von Phänomenen wie Massentourismus, Mobilität oder Identität mit Landschaften wichtig (z. B. SPOTTS und STYNES 1985; RIVLIN 1982; NOVICKI 1985; HAEGERSTRAND 1978).

Die Resultate der Los-Padres-Untersuchungen lassen sich auf dem Hintergrund der Betrachtungen von LEFF (1973) oder DIBBLE (1962), wonach Leute in verschiedenen Berufen unterschiedliche Ziele, Ideen, Interessen und Ideologien entwickeln, interpretieren. Die Resultate der empirischen Untersuchungen zeigen auf, daß die Tendenz besteht, Umweltwissen aktivitäts- und damit berufsspezifisch zu aggregieren.

Die Kontinuität und damit Ansätze zur Generalisierung liegen in der Typisierung routinemäßiger Abläufe (Aktivitätsräume, Tätigkeiten), durch die Umweltwissen produziert wird. Durch unterschiedliche Routinetätigkeiten bilden sich unterschiedliche Aktivitätsräume und in der Folge unterschiedliches Umweltwissen heran, was sich wiederum auf Handlungsbedingungen auswirkt (BREGMAN 1977).

DOUGHERTY und KELLER (1982) sind in ihren ethnolinguistischen Studien ebenfalls zum Schluß gekommen, daß Klassifikationen realer Objekte oftmals nur auf dem Hintergrund der zugrunde liegenden Tätigkeiten und Arbeitsabläufe verständlich sind. Tätigkeiten bilden das Grundmuster bei der Sammlung von Wissen (Klassifikationen, Sprache, Wahrnehmungsräume) über die gegenständliche Welt. Klassifikationssysteme sind im sozialen Kontext unterschiedlicher Produktionsweisen zu verstehen (vgl. ELLEN und REASON 1979).

Interessant ist dabei, wie bedeutsam die anthropogenen Elemente (z. B. Infrastrukturen oder Landbesitzmuster) für die Interpretation naturnaher Landschaften sind. Die generellen Ergebnisse der Arbeiten von APPLEYARD (1979) oder EDNEY (1976), die auf die Bedeutung von Symbolen in der Umweltkognition hingewiesen haben, werden bestätigt.

Zum Teil wird aus diesen Ergebnissen das Bedürfnis sichtbar, die Umwelt mit Bedeutungen zu belegen und sich an anthropogenen Elementen zu orientieren. Bereits KAPLAN (1976) hat darauf hingewiesen, daß in naturnahen Landschaften oftmals die nichtnatürlichen Objekte die deutlichsten Orientierungspunkte darstellen.

\section{Schlußfolgerungen}

BERGER und LUCKMANN (1966) haben sich aus handlungstheoretischer Sicht mit der sozialen Konstruktion von Realität beschäftigt und insbesondere auf die Bedeutung einer Soziologie von Wissen für die Soziologie hin- gewiesen. Die Resultate der Los-Padres-Studie weisen auf die Bedeutung dieser Interpretation hin.

Bis in die Mitte dieses Jahrhunderts bildete Umweltwissen, das aus direkter Erfahrung gewonnen wurde, das einzige verfügbare Wissen über naturnahe Landschaften. Mit dieser Studie haben wir einige Charakteristiken des «indigenous» Umweltwissen aufgezeigt, das in Planungsprozessen zunehmend beachtet wird (z. B. NOWICKI 1985).

Dieses «indigenous knowledge» wird zunehmend durch Umweltwissen, das dank modernen Methoden vor allem von staatlicher Seite und Wissenschaft großräumig systematisch gesammelt wird (z. B. Fernerkundung, Inventarisierung, Herstellung thematischer Karten) überlagert. In vielen Ländern steht dieser Prozeß der systematischen Datengewinnung erst am Anfang. Die sozialen Bedingungen dieses Prozesses werden selten thematisiert.

Die empirische Studie in Los Padres National Forest bringt Evidenz, daß die Akkumulation von Wissen durch Individuen - solche, die naturnahe Landschaften während ihrer Arbeit oder aber auf der Suche nach Erholung erfahren - nicht subjektiv ist, sondern sozialen Bedingungen und damit der Sozialisierung unterworfen ist.

Wichtige Bedingungen sind:

- Charakteristiken des Aktivitätsraumes

- Motive, Handlungen in der Landschaft

Beide Bedingungen sind sozialem Wandel unterworfen. Sie sind zum Beispiel zwischen traditionellen und modernen Gesellschaften grundsätzlich verschieden. Diesen sozialen Bedingungen der Akkumulation von Umweltwissen ist in Wissenschaft und Praxis (z. B. Entwicklungszusammenarbeit) vermehrt Beachtung zu schenken (vgl. auch EVANS 1980).

\section{Literatur}

APPLEYARD, D. (1970): Styles and methods of structuring a city. In: Environm. and Behav., 100-116.

APPLEYARD, D. (1979): The environment as social symbol: within a theory of environmental action and perception. In: Journ. of the Americ. Plann. Assoc., Vol. 45, Nr. 2, 143-153.

BARONI, M. R. et al. (1980): Memory for natural settings: the role of diffuse and focused attention. In: Perceptual and Motor Skills, Vol. 51, 883-889.

BECK, R. J., WOOD, D. (1976): Cognitive transformation of information from urban geographic fields of mental maps. In: Environm. and Behav., Vol. 8, Nr. 2, 199-238.

BERGER, P. L., LUCKMANN, T. (1986): The social construction of reality: a treatise in the sociology of knowledge. Doubleday Inc., New York.

BERRY, J.W. (1976): Human ecology and cognitive style. John Wiley Sons, New York.

BLAUT, J. M. et al. (1970): Environmental mapping in young children. In: Environm. and Behav., Vol. 2, Nr. 3, 335-349.

BREGMAN, A. S. (1977): Perception and behavior as composition of ideals. In: cognit. psych., Vol. 9, 250-292. 
CARR, S., SCHISSLER, D. (1969): The city as a trip: perceptual selection and memory in the view from the road. In: Environm. and Behav., Vol. 1, Nr. 1, 7-36.

CHAFE, W. L. (1977): The recall and verbalization of past experience. In: Current issues in linguistic theory (Herausgeber R. W. Cole), Indiana Univ. Press, Bloomington, 215-246.

DIBBLE, V. K. (1962): Occupations and ideologies. In:Americ. Journ. of sociology, LCVIII, 229-241.

DOUGHERTY, J.W.D., KELLER, C. M. (1982): Taskonomy: a practical approach to knowledge structures. In: Americ. Ethnol., Vol. 9, Nr. 4, 763-774.

EDNEY, J. J. (1976): The psychological role of property rights in human behavior. In: Environm. and Plann. A, Vol. 8, 811-822.

ELLEN, R. F., REASON, D. (1979): Classifications in their social context. Academic Press, London.

EVANS, G. W. (1980): Environmental Cognition. In: Psych. Bull., Vol. 88, Nr. 2, 259-287.

GOLLEDGE, R. G. et al. (1985): A conceptual model and empiricial analysis of children's acquisition of spatial knowledge. In: Journ. of environm. psych. Vol. 5, 125-152.

GOSSEN, G.H. (1974): Another look at world view: aerial photography and chamula cosmology. In: aerial photography and anthropological field research (Herausgeber: E. Z. Vogt); Harvard Univ. Press, Cambridge, 112-124.

HAEGERSTRAND, T. (1978): Survival and arena: on the lifehistory of individuals in relation to their geographical environment. In: Timing space and spacing time (Herausgeber T. Carlstein et al.), Vol. 2, London, 122-145.

JAMES, W. (1909): A pluralistic universe. Longmans Green, New York.
KAPLAN, R. (1976): Way-finding in the natural environment. In: environmental knowing (Herausgeber G. T. Moore und R. G. Golledge), Stroudsburg, 46-57.

KAPLAN, S., KAPLAN, R. (1982): Cognition and environment: functioning in an uncertain world. Praeger, New York.

LEFF, H. S. (1973): Construing the physical environment: differences between environmental professionals and lay persons. In: EDRA, Proc. 4th. conf., 284-296.

MOORE, G.T., GOLLEDGE, R.G. (1976): Environmental knowing: theories, research and methods. Dowden Hutchinson Ross Inc., Stroudsburg.

NOWICKI, P. (1985): Cultural ecology and management of natural resources. In: Culture an Conservation: the human dimension in planning (Herausgeber J.A. McNeely und D. Pritt), Croom Helm, London, 269-282.

RELPH, E. (1976): Place and placelessness. Croomhelm, London.

RIVLIN, L.G. (1982): Group membership and place meanings in an urban neighborhood. In: Journ. of sociol. issues, Vol. 38, Nr. 3, 75-93.

SAAB, Z. et al. (1984): Memory for places: role of schematarelated expectancy and saliency in recall and recognition. In: Psych. reports, Vol. 54, 607-610.

SPOTTS, D. M., STYNES, D. J. (1985): Measuring the public's familiarity with recreation areas. In: Journ. of leisure research, Vol. 17, Nr. 4, 253-265

WOHLWILL, J. F. (1976): Searching for the environment in environmental cognition research. In: MOORE und GOLLEDGE (oben zitiert, 385-392). 